\title{
Cloud-Native Repositories for Big Scientific Data
}

Ryan Abernathey $^{1}$, Tom Augspurger ${ }^{1}$, Anderson Banihirwe ${ }^{1}$, Charles C Blackmon-Luca ${ }^{1}$, Timothy J Crone ${ }^{1}$, Chelle L Gentemann ${ }^{1}$, Joseph J Hamman ${ }^{1}$, Naomi Henderson ${ }^{1}$, Chiara Lepore $^{1}$, Theo A Mccaie ${ }^{1}$, Niall H Robinson ${ }^{1}$, and Richard P Signell ${ }^{1}$

${ }^{1}$ Affiliation not available

January 19, 2021

\begin{abstract}
Scientific data has traditionally been distributed via downloads from data server to local computer. This way of working suffers from limitations as scientific datasets grow towards the petabyte scale. A "cloud-native data repository," as defined in this paper, offers several advantages over traditional data repositories-performance, reliability, cost-effectiveness, collaboration, reproducibility, creativity, downstream impacts, and access \& inclusion. These objectives motivate a set of best practices for cloud-native data repositories: analysis-ready data, cloud-optimized (ARCO) formats, and loose coupling with data-proximate computing. The Pangeo Project has developed a prototype implementation of these principles by using open-source scientific Python tools. By providing an ARCO data catalog together with on-demand, scalable distributed computing, Pangeo enables users to process big data at rates exceeding $10 \mathrm{~GB} / \mathrm{s}$. Several challenges must be resolved in order to realize cloud computing's full potential for scientific research, such as organizing funding, training users, and enforcing data privacy requirements.
\end{abstract}

\section{Hosted file}

Cloud_Native_Repositories_for_Big_Scientific_Data.pdf available at https://authorea.com/ users/372628/articles/490577-cloud-native-repositories-for-big-scientific-data 